\title{
Gestión e innovación educativa
}

Management and educational innovation

\author{
Evelyn Vargas Hernández \\ Directora Ejecutiva \\ Colegio Humanístico costarricense \\ Campus Omar Dengo. UNA, Heredia \\ evelyn.vargas.hernandez@una.cr
}

\section{Resumen}

La innovación pedagógica requiere de la incorporación de nuevas concepciones a los procesos ya existentes, para ello, la gestión administrativa debe cumplir con una serie de demandas que integran desde un ambiente laboral satisfactorio que permita atender las necesidades de los sujetos colaboradores de una institución educativa, hasta el seguimiento y la concepción curricular, la incorporación y desarrollo tecnológico, las necesidades e intereses de la población a la cual se dirige, la participación activa del educando, así como las competencias de los involucrados para que el proceso educativo este acorde con la realidad y se vuelva un aprendizaje significativo.

Palabras clave: Educación, innovación, gestión, habilidades, cambio, desafío, competitividad, calidad, competencia, cultura organizacional, clima laboral, motivación, currículo.

\section{Abstract}

Pedagogical innovation requires incorporating news conceptions to the already existing processes. To do so, administrative management must meet a number of demands integrating a satisfactory work environment that allows meeting the staff needs of an educational institution, the follow-up and curricular conception, the incorporation and technological development, needs and interests of the population to whom active participation of students and competences of people involved are 
addressed for the educational process to be in accordance with reality and to become a significant learning.

Keywords: Education, innovation, management, change, challenge, competitiveness, quality, competence, organizational culture, work environment, motivation, curriculum.

\section{Introducción}

Con base en el análisis de la realidad actual y las aspiraciones del desarrollo integral de la sociedad costarricense, el Ministerio de Educación Pública emite los lineamientos generales de acción en el marco de una política de empoderamiento de los centros educativos como eje vertebrador del mejoramiento de la calidad educativa. Según esto, se determina que la finalidad de la educación costarricense es la formación integral y plena del estudiantado que conforma el sistema en sus diversas modalidades, donde el pensamiento inspirador esencial es el de ideafuerza. Esta concepción del centro educativo de calidad es el eje de la educación costarricense, establecido por el Consejo Superior de Educación (2008).

En este sentido, los centros educativos de calidad deben impregnarse de esta idea central y regirse consecuentemente con las políticas educativas, las directrices que se emitan, los programas y las normativas que se dicten. Los programas, proyectos y acciones que se planteen deben estar evidenciados en las gestiones o actos administrativos que se realicen. De ahí que para el Consejo Superior de Educación de la República de Costa Rica (2008), la educación de calidad es un derecho fundamental, por lo cual se establece el carácter universal de esta política educativa y su aspiración intrínsecamente inclusiva; debido a que se considera esencial para el desarrollo de los seres humanos.

Para el logro de esto, la evaluación de los procesos institucionales juega un papel preponderante en la innovación de políticas de gestión que promuevan el mejoramiento de las acciones educativas. En este sentido, Valenzuela (2006) plantea que la evaluación es una parte esencial de cualquier proceso administrativo, e indica que está presente desde el momento mismo de la planeación, lo que permite diagnosticar el estado en el que se encuentra una institución. Esto permitirá brindar una oferta educativa que atienda las necesidades y aspiraciones sociales, en general, la atención a la diversidad, proporcionando oportunidades 
para el desarrollo de potencialidades, con variedad de estilos de aprendizaje y con condiciones sociales y económicas muy diversas.

\section{Innovación educativa}

Entre las reflexiones expuestas en el Primer Ideario Costarricense del siglo XXI (2010), encontramos que las transformaciones económicas, políticas, sociales, culturales y educativas, resultantes del mundo globalizado de hoy, también van alcanzando ineludiblemente el campo educativo (p. 605). En este sentido, las innovaciones pedagógicas están relacionadas con la idea de introducir nuevas concepciones a los procesos ya existentes, para lo cual la Comisión de Naciones Unidas Para el Desarrollo (1999), indica que en los países latinoamericanos existe un semillero de innovaciones pedagógicas. Esta tradición innovadora tiene su origen en el desarrollo de programas o proyectos "piloto" cuyos denominadores comunes son la imaginación, la creatividad y la voluntad de encontrar calidad en medio de las limitaciones de los sistemas tradicionales de educación.

Por medio de estos procesos se trata de modificar la clásica relación docenteestudiante, de aprovechar la dinámica de conocimientos, experiencias, contextos e interacciones que ofrecen muy distintos actores del proceso de enseñanza aprendizaje. Ruiz (2007) indica que la necesidad de cambiar, en la búsqueda de la innovación, es un proceso al que se enfrenta todo ser humano en algún momento de su vida; en ese sentido, las organizaciones educativas o de cualquier otra índole, se enfrentan a este proceso. Así, los cambios implican una serie de elementos que repercuten directamente en la cultura organizacional (tema que se desarrollará más adelante).

Por otra parte, Martín (2001) relaciona el proceso de innovación con la gestión de cambio, en la cual intervienen habilidades y conocimientos necesarios como adaptación, referido a la capacidad de acomodarse a los entornos cambiantes; orientación al cambio, entendida como la capacidad para estimular la introducción de novedades, las cuales pueden ser tecnológicas, organizativas o de otra índole; y multifuncionalidad, como la capacidad de desempeño en distintas áreas del trabajo. Todas ellas aunadas al dominio de técnicas de creatividad aplicadas a la innovación $\mathrm{y}$, particularmente, en el caso de los centros educativos al proceso de prácticas de planeación, desarrollo del currículo y a los procesos de mediación pedagógica. 


\section{Prácticas educativas innovadoras}

La educación es concebida como una actividad que debe ser abordada por los sujetos facilitadores o mediadores (educadores) desde un enfoque holístico e integral, involucrando los conocimientos del ser humano desde los diversos enfoques del saber y promoviendo en el estudiantado el análisis de su realidad. Para el logro de estos, las prácticas educativas innovadoras tienen que ver con el desarrollo de las habilidades y la autonomía desde un aspecto sistémico del conocimiento, con compromiso social y humano; aplicando aspectos metodológicos y pedagógicos para el desarrollo de competencias y manejo de habilidades blandas.

Tal como lo mencionan Fullan y Stiegelbauer (1997), para que una innovación sea eficaz debe ser multidimensional e incentivar a cambios acordes con los valores educativos, para lo cual es necesario alterar las convicciones de los individuos, desafiando los principios esenciales que estos poseen, en procura de satisfacer una necesidad determinada con mayor eficiencia que las prácticas existentes. Por esto, para que puedan desarrollarse prácticas educativas innovadoras, deben promoverse cambios en el proceso educativo. La innovación, dentro en estas prácticas, es un factor clave dentro del concepto de competitividad, por lo que es inherente un aprendizaje significativo en los estudiantes, algunas de estas pueden ser a través de la aplicación en el uso de las TICS (tecnologías de la innovación y la información).

Para que tanto docentes como estudiantes desarrollen nuevas competencias que les permitan aprovechar las herramientas tecnológicas funcionales para modelar su proyecto de vida, abrirse nuevas oportunidades de desarrollo laboral en la sociedad actual, aumentar la motivación para el autoaprendizaje y la exploración, y como estrategia para disminuir la deserción escolar, Rivera (2007) considera que:

El proceso de innovación tecnológica es el principal impulsor de la competitividad para una organización y el proceso de vigilancia de estas tecnologías, nuevas o renovadas, a través de la inteligencia competitiva y tecnológica es una herramienta fundamental para soportar la definición de estrategias competitivas (p. 9).

De igual forma, las nuevas prácticas educativas deberán promover la integración de innovación, reflexión, criticidad, experiencia, contexto social y conocimiento del medio como factores motivacionales del aprendizaje significativo; donde el estudiantado asuma un rol preponderante de su formación, ampliando los criterios 
de acción educativa según las necesidades de la población y así disminuir la deserción escolar. Pues, en múltiples investigaciones, se ha demostrado que la motivación del estudiantado, como fuente de aprendizaje, ha beneficiado el desempeño académico y ha incrementado las calificaciones y promedios, el ambiente de trabajo en el aula se vuelve más activo y estimula la retroalimentación entre docentes- estudiantes y estudiantes- estudiantes.

Por otra parte, Arulchelvan y Viswanathan (2006) expresan que el uso adecuado de apoyos tecnológicos permite una enseñanza más práctica y funcional, ya que se puede brindar el contenido con ejemplos claros y aplicado a la realidad; lo cual genera un mayor grado de atención en estudiantes y, por lo tanto, un aprendizaje significativo, propósito de las prácticas educativas innovadoras.

\section{Prácticas de planeación}

Como parte de nuevas tendencias a nivel internacional en la educación pública, las universidades han reformulado sus prácticas de planeación y manejo organizacional en términos de cierta flexibilidad, adaptabilidad y eficiencia, principalmente debido a las limitantes educativas y a las restricciones financieras por las que atraviesan. El Estado de la Nación (2001) indica que, dentro de una estrategia de desarrollo humano, la sociedad civil y el Estado deben ser garantes del libre acceso a la educación y a la capacitación, para lo cual la planeación debe ser considerada como una disciplina con amplia y decisiva implicación para el campo de la educación. Para ello, las instituciones de educación deben preocuparse por atender las necesidades de cambio de la sociedad, mediante la definición y redefinición de su misión social y estructura organizacional.

En este sentido, a lo interno de las instituciones educativas se lleva a cabo un conjunto de procesos de planeación institucional que constituyen un elemento inseparable de la función de gestión, toda vez que permite conducir a los centros educativos hacia el cumplimiento de su misión ante diversos escenarios, desde el eje central de una educación de calidad. Gregory Lozier, mencionado por Ruiz (2007), indica que la planeación es responsable de responder dos preguntas: ¿hacia dónde quiere ir la institución?, ¿cómo llegará ahí?, con estas preguntas el autor motiva al público lector a reflexionar acerca de la transformación institucional como un ámbito de suma importancia para la planeación, en la medida en que el cambio de los individuos y de las estructuras organizativas en la sociedad son parte del cambio social que refiere la participación de quienes planifican de la educación. 
En muy pocos casos se reportan en la bibliografía los procesos de reflexión crítica y de direccionalidad de la acción creativa que los sujetos docentes emprenden en los trabajos de planeación educativa. De esta forma, se invisibilidad las tareas sistémicas de dirección y aplicación de políticas operativas institucionales que incluyen el análisis del futuro de las decisiones actuales. Asimismo, existen limitados reportes sobre marcos metodológicos estratégicos que refieran el conocimiento y participación de los individuos para contribuir al cambio institucional como parte de procesos de desarrollo organizacional.

Por ello, la planeación es una de las actividades características en las instituciones educativas contemporáneas, y se vuelve cada día más necesaria, dada la velocidad en el acontecer de los fenómenos económicos, políticos, sociales y tecnológicos. Para Ruíz (2007), esta se entiende como planeación estratégica de calidad y está conformada por tres procesos: 1. Planeación normativa: incluye el desarrollo de la misión, visión y los valores institucionales. 2. Planeación estratégica: integrada por las estrategias que surgen de la misión, visión y los escenarios. 3. Planeación operativa: tiene que ver con los objetivos y los planes de acción, es donde sucede el cambio.

Así, la planeación es un elemento inseparable de la función de gestión organizacional, toda vez que permite conducir la administración institucional hacia el cumplimiento de su misión, objetivos y metas. La planeación va más allá de la simple espera de la ocurrencia de los eventos futuros; a la luz del diseño o visualización de escenarios esperados, esta implica la forma de modificar y mejorar estos escenarios.

La planeación es el proceso de establecer objetivos y escoger el medio más apropiado para su logro antes de emprender la acción; se anticipa a la toma de decisiones, lo cual consiste en un proceso de decidir antes de que se requiera la acción. Para Chen y Vargas (2007), los principios estratégicos enfatizan la apropiación local del proceso de planeación, la participación efectiva en todos los niveles y un alto compromiso. Esto destaca la importancia de lograr la convergencia y coherencia entre diferentes esquemas de planeación, mejorando la sinergia y la remoción de inconsistencias.

\section{Currículo}

El currículo promueve el alcance de las aspiraciones que la sociedad deposita en el proceso educativo, teniendo en cuenta los aspectos individuales, sociales y culturales. Chen y Vargas (2007) indican que el planteamiento de un currículo 
para la educación siempre ha sido tarea difícil y de gran responsabilidad para los sectores académicos. Por lo cual la administración del currículo es un proceso primordial dentro de la gestión educativa. En este sentido, el Informe del Estado de la Educación (2011) hace referencia a que el currículo debe estar acompañado de procesos de investigación, lo cual es un desafío central para mejorar la calidad educativa del sistema costarricense.

Por su parte, Bolaños y Molina (1996) plantean que en el caso del currículo el objeto de estudio son los procesos de enseñanza y aprendizaje, así como el contenido de estos. Sin embargo, especifican que, el personal docente no siempre es especialista del currículo, pero como guía del proceso enseñanza aprendizaje, tiene a cargo su ejecución. Por lo cual la labor docente en este proceso será trascendental para el logro de los objetivos propuestos.

\section{Gestión administrativa}

Los procesos de gestión administrativa buscan el aprovechamiento de las destrezas de sus sujetos colaboradores para el logro máximo de la organización y atención de problemas complejos, entre estos, las relacionadas con el éxito en el trabajo y en las relaciones interpersonales. Ante esto, Novais (2000) menciona que relacionarse bien en el trabajo es una ciencia y un arte. Ciencia que no todos aprenden a poner en práctica: "Arte porque requiere la sensibilidad de percibir lo que podemos y debemos hacer en las circunstancias más diversas" (p. 259).

Desde un enfoque humanista, se concibe a la persona como un ser integral, de mente y corazón, no como simples máquinas programadas para realizar un trabajo y nada más. El ser humano siente cuando lo estiman, aprecian e infunden simpatía; o, por el contrario, cuando lo apartan y desprecian alejándolo de las relaciones entre sus pares.

\section{Gestión en centros escolares}

Debido a los acelerados cambios que se han sucintado en los últimos años, cada día se hace más urgente el planteamiento de cambios educativos que estén acordes con las exigencias y demandas del contexto actual y las futuras generaciones. En este sentido, es necesaria una transformación de la gestión educativa tradicional a una cultura de gestión organizacional. Se requiere un cambio de mentalidades y actitudes que impliquen modificaciones a las estructuras organizacionales para 
dar paso a verdaderas comunidades de conocimiento en los centros escolares. Al respecto, Flores y Torres (2010) plantean la importancia de convertir a las escuelas en organizaciones reflexivas que tengan como meta el mejoramiento continuo.

A través de los años, los patrones culturales de las escuelas han permanecido casi inalterables en sus prácticas habituales. Marcelo y Estebaranz (1993, en Flores y Torres, 2010) señalan que hoy las escuelas mantienen las mismas estructuras, currículos, y organización desde hace varias décadas. Por esto, los entes que integran el proceso han aprendido con el paso de cada generación a reproducir patrones tradicionales y la ciudadanía, en general, no ha asumido una responsabilidad real ante las exigencias que plantea la sociedad actual.

Como parte de los nuevos diseños en la administración educativa, existe un amplio consenso en que los resultados de aprendizaje dependen de la calidad de la interacción y del proceso pedagógico que ocurre en los salones de clases, con base en las políticas institucionales que rigen esa relación. Alvariño (2000) indica que los resultados académicos se explican por diversos factores que pueden clasificarse en internos y externos. Los factores internos corresponden a los aspectos propios del centro educativo, es decir, insumos, capacitación, salario docente, gasto por estudiante, sistema de incentivos, liderazgo de los directores o directoras, etc. Por otra parte, los factores externos consisten en aspectos de comunidad, esto es, desempleo, pobreza, delincuencia y, por otra parte, la situación familiar, es decir, el ingreso, la escolaridad de los padres y madres, los afectos, la psicología del hogar, etc.

Por ende, asumir el mejoramiento de la calidad de la educación como una prioridad en el desarrollo de las políticas de cada país ha provocado consenso en los procesos de reforma y se considera que el elemento trascendente está vinculado con el cambio cultural de los centros educativos. El liderazgo distribuido representa un cambio que revoluciona la cultura administrativa de la escuela, desarrollando procesos de participación, democratización y corresponsabilidad de los resultados escolares, entendidos estos como el desarrollo de toda la comunidad escolar. De esta forma, al evaluar las prácticas educativas innovadoras, es imprescindible realizar una mirada a la gestión escolar que realiza la dirección del centro educativo y sus características, en búsqueda de condiciones particulares de liderazgo y de transformaciones que permitan asegurar la instalación de procesos de gestión educativa para el mejoramiento permanente. 
La temática de las relaciones humanas posee una estrecha relación con factores como: la personalidad, la comunicación y, sobre todo, con la autoestima de los individuos, ya que quien aprende a valorarse y autoestimarse, a pensar bien de sí y de las demás personas, cultiva una buena salud psíquica y excelentes relaciones. En ella interviene, de forma directa, el desarrollo interno de la cultura organizacional, el clima, la motivación y la satisfacción laboral.

\section{Cultura organizacional}

Cada organización posee una cultura, lo cual quiere decir que existe un sistema de significados que comparten sus miembros, de tal manera que unas personas pueden ser rígidas, y otras flexibles, hostiles o acogedoras, innovadoras o conservadoras, por ello no se pude generalizar, ya que cada una tiene su carácter singular y eso es lo que las hace diferentes a unas de otras. Robbins (2004) define la cultura organizacional como un sistema de significados compartidos por miembros de una organización, que la distinguen de otras. Este sistema es un conjunto de características básicas que valora la organización.

En toda institución existe un sistema de comportamiento organizacional, en el cual se incluyen la filosofía, los valores, la visión, la misión y los objetivos de la entidad, la calidad del liderazgo, la comunicación y la dinámica de los grupos; se combinan para crear una cultura en que las actitudes de su personal y los factores situacionales pueden generar el logro de los objetivos. La cultura organizacional es parte de un complejo sistema social, en donde predominan y se interrelacionan los valores, las costumbres, las creencias, tradiciones, formas de pensar de cada miembro involucrado. Según Davis y Newstrom (1993): “...es el conjunto de supuestos, convicciones, valores y normas que comparten los miembros de una organización" (p.153). Además, afirman que es creada por parte de las personas que conforman el equipo de trabajo, de manera consciente o inconsciente; lo cual se manifiesta en cada persona, pues, aunque la cultura no sea palpable o tangible puede influir de manera sobresaliente en cada individuo.

Por medio de la cultura organizacional se permite diferenciar a una institución de cualquier otra, lo cual facilita y promueve en cada uno de sus miembros la satisfacción y la motivación laboral. Si la cultura es adecuada, permite desarrollar las potencialidades del personal que puede visualizarla como un modelo a seguir. De esta forma, la gestión educativa tratará de promover alternativas organizacionales acordes con las políticas de la institución. 
Cabe destacar que ninguna cultura es mejor que otra, pues la multiculturalidad que en ella se evidencia está determinada por la misión, metas y las competencias de cada centro educativo. De igual manera, es relevante indicar que sus miembros tienen que adoptar, o al menos aceptar, los valores de la organización. Para trabajar en conjunto, de una manera eficaz, los individuos necesitan decidir colectivamente cómo distribuir poder, estatus y autoridad. Necesitan establecer un conocimiento compartido acerca de quién obtendrá las recompensas y las sanciones por tipos específicos de acciones.

Por ello que se puede considerar la cultura organizacional como un sistema muy complejo que comparten miembros de una institución educativa; así, esta indica la manera de comportarse y de cómo deben desarrollarse las funciones dentro de la entidad.

Por tanto, la cultura organizacional se caracteriza por ser un término de tipo descriptivo, pues los funcionarios y funcionarias son quienes la definen tal y como la perciben; está compuesta por características de relativa estabilidad, las cuales surgen con el paso de los años y se arraigan continuamente; donde prevalece la misión, la filosofía, el estilo de liderazgo, los criterios de contratación, la práctica de los ascensos, entre otros. Por ende, dicho factor es determinante en cualquier entidad, pues les da a los colaboradores y colaboradoras un sentido de pertenencia que les induce a sentirse a gusto y en correspondencia con el sitio de trabajo, aspecto indispensable cuando se hace referencia a dar y recibir calidad en el sistema educativo.

\section{Clima laboral}

Corresponde al ambiente que generan los miembros de una empresa, organización o institución donde laboran, donde se ven reflejados sus comportamientos, costumbres, valores y actitudes. De tal forma, que puede ser un clima agradable de confianza o de respeto; o por el contrario un clima hostil y frío. Al respecto, Garza (2000) apunta que: "Es el conjunto de valores y normas que rodean el ambiente, de tal manera que orientan las actitudes y conductas de los trabajadores de una empresa" (p. 89).

El clima laboral, a diferencia de la cultura organizacional, es más cambiante, ya que se refiere al ambiente interno, pero influenciado por agentes externos a esta. 
Sin embargo, no es correspondiente a los aspectos físicos o estructurales, sino que se correlaciona con los grados de motivación de sus participantes.

Además, este ambiente permite que se creen grupos, donde ocurren procesos dinámicos autogeneradores. En el ámbito educativo, el personal docente interactúa entre sí y con el estudiantado, estas interacciones desarrollan sentimientos favorables hacia colegas y hacia los grupos. A la vez, estos sentimientos vienen a ser la base de distintas actividades, muchas de las cuales no se habían especificado en la descripción de las funciones del profesional, por ejemplo, arreglos especiales para el almuerzo, participación en eventos extracurriculares, acompañamiento en giras, atención de situaciones emocionales, sentimentales o académicas, intercambio de tareas y deberes, entre otras.

La comprensión de lo que representa el clima dentro de cualquier institución faculta al personal encargado de la gestión a optar por un mejor desempeño del recurso humano, al influenciar aspectos personales de cada persona trabajadora, como la autoestima, la motivación y satisfacción; así como aquellos que se relacionan más con el medio en que se desenvuelven, tal como las relaciones interpersonales, la economía, la cultura organizacional, entre otros.

\section{Motivación laboral}

Generalmente, se emplea para referirse a las razones o motivos que explican nuestro comportamiento y el comportamiento de las demás personas. Alier, Briñol, Caballero y De la Corte (1999) consideran que la motivación es uno de los factores que determinan la satisfacción y el rendimiento laboral, saber cómo funciona y en qué modo es posible intervenir sobre ella puede resultar muy útil, tanto para el personal que desea conocer las razones de su comportamiento en el entorno de trabajo, como para el sujeto administrador o gestor institucional.

La motivación es entendida como los impulsos o necesidades humanas que incitan a la persona a desarrollar sus capacidades y a buscar continuamente su actualización. Según Reeve (1994): representan un proceso dinámico, más que un estado fijo o permanente; tienen puntos de declive y de crecimiento continuo, y pueden ser estimuladas por fuerzas intrínsecas, tales como la fatiga o la curiosidad, los intereses, las necesidades y las reacciones personales del comportamiento. Cuando las motivaciones provienen de factores intrínsecos 
se pueden crear conductas auto-reguladas, estas provienen de los intereses, las necesidades y las reacciones personales.

Al respecto Schermerhorn, et al. (2004) apuntan que, en su definición formal, la motivación se refiere a las fuerzas individuales que explican la dirección, nivel y persistencia del esfuerzo que una persona invierte en el trabajo. En este sentido, las personas están motivadas cuando muestran deseos de realización, de ambición y de promoción en el trabajo, en otras palabras, muestran buena disposición para asumir responsabilidades.

\section{Satisfacción laboral}

El ajuste al trabajo se define en términos de los esfuerzos que las personas realizan para mantener la correspondencia entre sus necesidades y los requerimientos del trabajo que están realizando. De esta manera, de acuerdo con Casullo, Cayssials, Fernández, Wasser, Arce y Álvarez (1994):

La satisfacción con el trabajo elegido es una función de la correspondencia entre el sistema de refuerzos que brinde el medio laboral y las necesidades individuales, siempre que las personas tengan las habilidades mínimas para cumplir con eficacia lo que requiere el trabajo en el que se desempeñan. El trabajo en sí mismo afecta a la satisfacción de los individuos, en este sentido el desempeño del trabajador dependerá de la actitud que este asuma ante la labor que debe ejecutar. De esta forma, Al igual que la motivación, la satisfacción laboral también es afectada por el sistema ambiental y la retroalimentación recibida contribuye a la satisfacción y ejercicio de los docentes.

Que un individuo se encuentre satisfecho con la labor que realiza depende de aspectos tales como: las expectativas referentes al puesto y labor que desempeña; la autoevaluación que cada persona trabajadora debe de realizar para poder determinar si lo que realiza está dentro de sus posibilidades físicas, psicológicas y emocionales. O sea, si las demás personas valoran y aprecian lo que se hace a través del trabajo; comparaciones sociales que se relacionan con el trabajo que desempeñan quienes le rodean; relación insumo-resultado, determinante en que lo que la organización da es equitativo con lo que su personal le da a ella: compromiso e ilusiones. 
Ahora bien, la satisfacción laboral que cada individuo experimente depende de su contexto, de las características que para esa persona sean importantes para sentirse a gusto con la labor que realiza y con lo que la organización le devuelve por ella. Además, debe ser una de las principales determinantes del comportamiento de sus colaboradores de una institución. De esta manera, Robbins (2004) agrega que: "Los empleados satisfechos se inclinan más a hablar positivamente de la organización, ayudarla, auxiliar a los demás y superar las expectativas normales de su puesto" (p. 98).

Cabe destacar que a las actitudes en los sujetos colaboradores no se les debe de restar importancia, pues en relación con las dimensiones antes expuestas, las actitudes que demuestren afectan directa o indirectamente en el comportamiento general de la institución. De esta manera, se rescata la interrelación que existe entre la satisfacción laboral con el desempeño que los trabajadores y trabajadoras ejercen en cada institución.

\section{Competencias}

El concepto de competencia implica ambigüedad en su significado, pues si a un sujeto docente se le preguntara sobre las competencias, posiblemente, de acuerdo con su especialidad pedagógica, daría una serie de respuestas disímiles. Mientras que, si esa misma pregunta se les hace a quienes se encargan del personal de diversas empresas, lo más seguro es que las opiniones no sean tan variadas, porque coinciden en señalar que ven reflejadas las competencias en la efectividad productiva y en la ejecución de las funciones laborales que les asignen a los trabajadores y trabajadoras.

A pesar de que cada uno de los grupos tiene la verdad en sus razones, ninguno de ellos es completamente neutral. El significado de competencia, para quienes tienen la responsabilidad de impulsarlas, está ligado a las experiencias particulares de quien arriesgue una definición, según lo expuesto por Maldonado (2001):

El docente las relaciona con el metalenguaje pedagógico; desde su diccionario educativo, establece asociaciones con sinónimos de capacidad, logro, realización, indicador $\mathrm{u}$ otras acepciones. El encargado de personal por su parte, establece asociaciones con el trabajo, tales como: producción, honradez, profesionalismo, eficiencia u otra serie de acepciones ligadas al rendimiento productivo. (p.132) 
Según el autor antes mencionado, la disertación sobre el tema tiene dos referentes: el primero, el de las competencias básicas, que le corresponden en especial al sistema educativo, estructurado desde una plataforma lingüístico-comunicativa; y el segundo, el de las competencias laborales o profesionales que le corresponde directamente a la educación orientada a la formación de trabajadores y trabajadoras, donde se toman como referente modelos estructurales funcionalistas, propios de la producción empresarial. Asimismo, es necesario establecer un concepto de competencia que se adapte al entorno laboral. De esta manera, Maldonado (2001), a partir del Consejo Nacional de Cualificaciones (1994), la define de la siguiente forma:

Capacidad de realizar las actividades correspondientes a una profesión conforme a los niveles esperados en el empleo. El concepto incluye también la capacidad de trasferir destrezas y conocimientos a nuevas situaciones dentro del área profesional y, más allá de ésta, a profesiones afines. Esta flexibilidad suele implicar un nivel de destrezas y conocimientos mayor a lo habitual, incluso entre trabajadores con experiencia. (p. 34)

Es decir, un sujeto trabajador es competente para desempeñarse en la estructura productiva en tanto demuestre capacidad de análisis, de síntesis, sepa tomar decisiones, manipule los utensilios con los que debe trabajar, pueda trabajar en equipo, comprenda los procesos tecnológicos y, sobre todo, desarrolle capacidad productiva en el marco de la estructura empresarial. Mertens (1996, citado por Maldonado, 2001) parte de las experiencias que los sectores empresariales tienen sobre su personal, estableciendo a partir de esta demanda el papel de la formación profesional y de la producción. Sus aportes se orientan, en esta etapa, a la identificación de los requerimientos laborales que los entes patronales demandan sobre los empleados y empleadas. Es decir, su propósito es la instauración de modelos de formación basada en competencias laborales.

De esta manera, se hace notar que para quien contrata el personal resultan vitales las competencias que permitan, en el desempeño de trabajo, la capacidad de transferir los conocimientos, habilidades o destrezas asociadas al ejercicio de una labor productiva, así como la capacidad de resolver problemas cotidianos y extraordinarios asociados a sus funciones laborales.

El conocer las competencias personales contribuye en el entendimiento de la propia realidad y los aspectos desencadenantes de ella, en los diversos espacios. De ahí la necesidad de establecer los niveles de competencia necesarios en la 
formación docente, para poder, por medio de estos, establecer el perfil académico en el marco de un centro educativo innovador, en cuyos procesos de gestión administrativos se evidencie el desarrollo de nuevas políticas educativas.

\section{Competencias docentes}

El personal docente es el factor clave para cualquier innovación educativa, es él quien la construye y la implementa. Por eso se le debe prestar especial atención, y se debe sensibilizar al cambio, para que muestre disposición y flexibilidad, de ahí la necesidad de que posea una serie de competencias especiales que le permitan este ajuste.

De la Torre (1995, citado por ANUIES y UPN 2004) menciona que el papel docente, actualmente se va a ver transformado; pasó de ser transmisor de información y debe asumirse como profesional activo que va a mejorar su práctica educativa con la innovación y la investigación. El personal docente, entonces, se va a ver beneficiado con el desarrollo de habilidades de conocimiento que le permitan modificar estrategias de enseñanza aprendizaje utilizando las TIC y otros recursos didácticos de apoyo. Así mismo, como mencionan ANUIES y UPN (2004), se va a beneficiar, también, adquiriendo un nuevo rol que le va a permitir: anticipar la pertinencia del aprendizaje, evaluar competencias, crear ambientes para aprendizaje significativos, generar nuevos conocimientos, formar a sus estudiantes en la búsqueda, síntesis, análisis, favorecer la autonomía, creatividad, actitud crítica y confianza de sus estudiantes.

Al mismo tiempo, el personal docente es un facilitador del proceso educativo, lo cual implica que debe avocarse al desarrollo de sus propias competencias, entre estas: desarrollo de valores, promover la conciencia social, extender el amor por el arte, la estética y la cultura, propiciar un compromiso social entre sus educandos, promocionar la investigación y estimular la construcción de nuevos conocimientos.

Tal como lo indica Flores y Torres (2010), las innovaciones en educación están dirigidas por los educadores y educadoras, quienes deben ser agentes de cambio. Necesitamos realizar investigación de vanguardia en un campo especifico en función del estado del conocimiento y a nivel internacional, pero esto no debe quedar ahí, se debe divulgar lo que se conoce en el ámbito de la discusión pública 
sobre la educación y colaborar activamente en la innovación de los programas educativos y planeamientos de clase.

La gestión administrativa debe promover la capacitación del personal, lo cual es básico en la implementación de proyectos de innovación, esta puede ser auto o mutua capacitación, pues sin la preparación y sensibilización del profesional para el uso adecuado de los recursos de apoyo se podría correr el riesgo de que no se tenga el éxito esperado. Cada docente debe batallar con muchos factores, y debe superar muchas crisis internas para poder ser capaz de promover el cambio. Más aún, tiende a aumentar la dependencia de los maestros y maestras hacia el conocimiento práctico necesario para las tareas diarias, lo que excluye las fuentes de conocimiento que están más allá de su experiencia en el salón de clases.

Por lo cual, se hace imprescindible promover un cambio de visión educativa, donde puede tener la oportunidad de adquirir nuevas habilidades y destrezas laborales, tener acceso a un trabajo para dar apoyo económico a su familia y desarrollar las competencias necesarias para modelar su proyecto de vida.

\section{Considerandos finales}

De esta forma, se concluye que la gestión administrativa innovadora debe cumplir con una serie de demandas, entre las cuales se encuentran las concepciones desarrolladas en el presente artículo, las cuales plantean la búsqueda de un ambiente laboral satisfactorio que permita atender las necesidades de colaboradores y colaboradoras de una institución educativa desde las diversas facetas humanas, las cuales van desde motivación intrínseca y extrínseca hasta ambientes de trabajo idóneos y de tendencia humanista. De igual forma, es imprescindible el seguimiento y concepción curricular para que se cumpla con los contenidos académicos necesarios a cada programa educativo, el cual debe ser enriquecido con las experiencias, necesidades e intereses de la población a la cual se dirige. Sin dejar de lado la participación activa del estudiantado, para que el proceso educativo sea acorde con la realidad y se vuelva un aprendizaje significativo.

El desarrollo de una visión compartida para el planeamiento institucional se vuelve perentorio, tal como lo destaca Senge (2007), si una idea sobre liderazgo ha inspirado a las organizaciones durante muchos años es la capacidad para compartir una imagen del futuro que se procura llegar a obtener. Para lo cual 
son necesarias las implementaciones de sistemas transformadores que atiendan las prácticas educativas de planeación, tanto administrativas como curriculares, y donde el enfoque de formación y competencias de su personal asume un rol decisivo en la toma de decisiones y en la gestión de las instituciones de educación que promueven una educación de calidad.

Adicional a la formación académica, cada centro educativo debe propiciar modelos centrados en el desarrollo integral de sus estudiantes, donde se consideren sus cualidades y sus experiencias, que se les estimule a conocerse y a decidir lo que quieren llegar a ser. La libertad de escoger ha sido un pilar fundamental en el quehacer de las instituciones innovadoras, al estimular el desarrollo de una personalidad creativa, consciente de sus fortalezas y debilidades, pero segura al enfrentar los retos y dificultades del momento actual. De esta forma, para el logro de instituciones educativas innovadoras es necesario que exista una relación intrínseca y cooperativa entre los procesos de gestión administrativa y su articulación con las prácticas educativas que asume el personal docente, administrativo, el estudiantado y las familias; los cuales, por las consideraciones mismas, deben poseer un perfil especifico con competencias determinadas por la gestión de cada centro educativo.

\section{Referencias}

Alier, E., Briñol, P., Caballero, A. y De la Corte, L. (1999). Relaciones en el entorno de trabajo. España. Editorial PARANINFO.

Alvariño, C. et al (2000). Gestión escolar: un estado del arte de la literatura. Revista Paideia, 29, pp. 15-43

ANUIES, \& UPN. (2004). Documento estratégico para la innovación en la educación superior. México: ANUIES.

Arulchelvan, S., \& Viswanathan, D. (2006). Pattern of usage of various electronic media by higher education students. International Journal of Education and Development using Information and Communication Technology. Bridgetown, 2(4).

Bolaños, G. y Molina, Z. (1996). Introducción al currículo (1era Ed.). San José, C.R.: EUNED. (1990) 
Casullo, M., Cayssials, A., Fernández, M., Wasser, L., Arce, J. y Álvarez, L. (1994). Proyecto de vida y decisión vocacional. Buenos Aires, Argentina: Paidós.

Chen, E. y Vargas, I. (2007). Administración de recursos institucionales en centros educativos. Costa Rica: EUNA.

Consejo Superior de Educación. (2008). El centro educativo de calidad como eje de la educación costarricense. República de Costa Rica.

Comisión de las Naciones Unidas para el Desarrollo. (1999). Los retos educativos del futuro. Estado de la educación en América Latina y el Caribe. Costa Rica: Guilá.

Davis, K. y Newstrom, J. (1993). Comportamiento humano en el trabajo. México: McGraw-Hill Interamericana.

Estado de la Nación en Desarrollo Humano Sostenible. (2001). Proyecto Estado de la Nación: Módulo para educación secundaria. San José, Costa Rica.

Estado de la Nación en Desarrollo Humano Sostenible. (2011). Proyecto Estado de la Educación: Consejo Nacional de Rectores (3era Ed.). San José, Costa Rica.

Flores F. M, Torres H. M. (2010). La organización como escuela de conocimiento. México: Trillas.

Fullan, Michael G. \& Stiegelbauer, Suzanne. (1997). El cambio educativo: Guía de planeación para maestros. México: Trillas.

Garza, T. (2000). Administración contemporánea. Distrito Federal, México: McGraw-Hill.

Maldonado, G. (2001). Las competencias, una opción de vida. Metodología para el diseño curricular. Bogotá, Colombia: Ediciones ECOE.

Martín, E. (2001). Gestión de instituciones educativas inteligentes ( $2^{\mathrm{a}}$ Ed.). México: McGraw-Hill Interamericana.

Novais de Germano. (2000). Cómo obtener éxito en las relaciones humanas. Bogotá, Colombia: Instituto Misionero Hijas de San Pablo. 
Primer Ideario Costarricense del Siglo XXI. (2010). Programa de publicaciones e impresiones de la Universidad Nacional. Heredia, C. R.: Editorial.

Reeve, J. (1994). Motivación y emoción. Madrid, España: McGraw-Hill.

Rivera González José. (2007). Propuesta de inteligencia competitiva y tecnológica para la generación de estrategias de innovación futura. ITESM-Campus Monterrey-EGADE. Biblioteca Digital T1743.H37.2007b.

Robbins, S. (2004). Comportamiento organizacional. Juárez, México: Editorial Pearson Prentice Hall.

Ruiz, M. (2007). Sistema de planificación para instituciones educativas. México: Trillas.

Schermerhorn, J., Hunt, J. y Osborn, R. (2004). Comportamiento organizacional. Distrito Federal, México: Limusa Wiley.

Senge. P. (2007). La quinta disciplina: El arte y la práctica de la organización abierta al aprendizaje ( $2^{\mathrm{a}}$ Ed.). Buenos Aires: Granica.

Valenzuela, J. (2006). Evaluación de instituciones educativas. México: Trillas y ITESM. 